\title{
Four Theses About Qualia and Matter: From Quality to Structure, from Structure to Functions
}

\author{
Aleksandr Vladimirovich Zhuravlev \\ Neurogenetics Laboratory, Pavlov Institute of Physiology, Russian Academy of Sciences, St. Petersburg, Russia \\ Email address: \\ beneor@mail.ru \\ To cite this article: \\ Aleksandr Vladimirovich Zhuravlev. Four Theses About Qualia and Matter: From Quality to Structure, from Structure to Functions. \\ International Journal of Philosophy. Vol. 5, No. 3, 2017, pp. 23-29. doi: 10.11648/j.ijp.20170503.11
}

Received: April 2, 2017; Accepted: April 18, 2017; Published: June 1, 2017

\begin{abstract}
The mind-body problem is considered here in terms of quality and structure, which are believed to be intimately related. Both qualia, the elements of phenomenal consciousness, and their material causes possess specific structure of inner relations founded by their qualitative nature. This seems to define their functions in the process of cognition. Qualia are intrinsically organized as a complex multifold system, where the general relations, forms, and properties are represented as quasi-separated phenomena. Reflected by the structure of the brain physical processes, they may assist to conceive the general laws and regularities of nature, thereby helping a subject to optimize his behavioral strategy.
\end{abstract}

Keywords: Qualia, Matter, Mind-Body Problem, "the Hard” Problem of Consciousness

\section{Introduction}

When we speak about consciousness, there are two main classes of questions which were named by David Chalmers as the "easy" and "the hard" problems. "The easy" problem of consciousness relates to the complex organization of the cognitive processes in brain, such as the process of attention, categorization, deliberate control of behavior, etc. All of them apply to the information processing and are successfully studied by science. "The hard" problem relates to the so-called conscious experience or phenomenal consciousness: "Why doesn't all this information-processing go on in 'the dark', free of any inner feel? [2]". The question is about qualia (singular: quale), the structural units of phenomenal reality known to us directly from our experience.

In modern philosophy, plenty of words have been said about qualia, regarding their subjective evidence, specific qualitative nature and attributes, their mode of existence "from the first-person point of view [2]", irreducibility to brain processes, both physical and functional, etc. Even nowadays, the mind-body problem, or the problem of "explanatory gap" between physical and qualitative aspects of mental states, is widely discussed [3]. I would like to address the reader to the works of famous mind philosophers, such as John Searle, Daniel Dennett, Frank
Jackson, David Chalmers, and many others. Some of them are focused on the specificity of phenomenal qualia, while the others, like Dennett, are rather interested in the evolutionary processes that have given rise to human mind [4].

What I shall focus on in this work is "the hard" problem of consciousness: why do we need qualia to think, analyze, and draw conclusions about something in the world? The existence of qualia, as I try to show here, is evident, moreover, directly we do not know anything but qualia and their structure. In addition, there is no way to solve "the hard" problem of consciousness disregarding qualia themselves and their specific qualitative nature. The discussion about the physical and physiological substrates of qualia, their ontological nature, and the possibility of philosophical zombie existence sidetrack us from the main goal - to find a connection between qualitative nature of consciousness and its possible functions. Function can be defined as an influence on some events, their passing and structural modifications. Thus, my goal is to find interrelation between qualitative and structural aspects of qualia, which inner architecture possibly serves as a framework for some physical events in brain. 


\section{Four Theses About Qualia and Matter}

\subsection{Qualia are Elements of Conscious Experience Characterized by the Inner Intentionality}

Jackson gives the following definition of qualia: they are "certain features of the bodily sensations especially, but also of certain perceptual experiences, which no amount of purely physical information includes [5]". Not only the raw senses, such as blue color, acrid taste, and loud sound, can be qualified as qualia, but everything that exists inseparably from the observer and his consciousness, or, speaking by Searle's language, from the "first-person point of view".

For instance, such estimations as "small and big" or "right and left" require a subjective observer: it is impossible to imagine an object innerly "big" or innerly "left", without any relation to subject. This should not be mixed up with "biggersmaller" and other relations, which are not founded by subjects' point of view and have the wholly mathematical basis. The same differences are between the extension as an abstract mathematical multitude with continuum order of its members and the extension as our specific sensual mode to perceive spatial forms and objects. To speak about pure sensations, blue is more or less similar to cyan and violet, also, blue is a "cold" color. Hence, the sensations of different modalities form a complex system of relations inseparable from their qualitative nature. Thus, we can say, qualia are elementary subjective phenomena representing some structure of mathematical relations.

There are two main questions concerning the previously mentioned:

1. What does it mean to be known from "the first-person point of view"?

2. How is it possible for us to know anything besides our qualia?

It's rather tricky to define what phenomenal, or ontologically subjective, or obvious is inherently. The simplest way to express it is - "nothing to argue about". However, it's well known how many discussions about qualia and the very fact of their reality are ongoing [3], [6]. Some philosophers consider the phenomenal qualities to be only specific behavioral dispositions [7]. Whenever I think about quale, e.g. red color, everything in it can be described in the language of behavior: to recollect pink and orange, to expect something hot and burning, to stop the car before the light signal, and so on. The very qualities of qualia seem to dissolve in the complex system of relations and intentions. Dennett even considered all the people to be "zombies" [8], as, from the behavioristic point of view, qualia are some kind of phantom or mistake. Nevertheless, it is impossible to abnegate qualia, if we identify them with what is directly evident and apparent, without any reference to their effectiveness or ontological nature.

Then - what is evident and to whom is it evident? Subjective consciousness is often considered to have two defining features: phenomenology or self-presentation immediacy, and intentionality or feature to represent something other in mind or external world [9]. These properties are thought to be either intimately related (inseparatism) or independent of one another (separatism). For instance, fear represents something terrible, the multitude of specific events in mind may represent the thought of common law, but the sense of thirst or sadness apparently represents nothing. The quale of sadness is a simple phenomenon, and it is tricky to separate the object of sadness from the subject suffering sadness. The very opposition of subject and object of view seems to be inherent only to complex mental phenomena structured by intentionality.

Even a simple quale, however, seems to possess some kind of intentionality. Imagine the world A where only the pure sense of blue color exists and nothing more. Also, imagine the world $\mathrm{B}$ where one and only one physical event exists without any observer. What is the main structural difference between $\mathrm{A}$ and $\mathrm{B}$ ? If we say that $\mathrm{A}$ is innerly observed by the "first-person" and B is not, this returns us to the principle question: are there any differences between to be a simple quale and to experience a simple quale? However, if anything is added to blue, i.e. geometric form of a blue object, both the intentionality and the subject-object division immediately appear: blue sense starts to represent some spatial object. This property is fundamental for qualia: their specific mode of organization is to represent each other or some abstract relations. Being a part of the integral subjective world, sadness is inevitably inbuilt in the complex system of mental associations and dispositions, encouraging the subject to seek some consolation. Thus, we can say, all qualia as subjective phenomena possess the intentionality in the "latent form" or the inner intentionality.

Now it is time to answer the second question. All that we know becomes familiar to us in the form of qualia, including specific qualia of abstract thinking. At the same time, some of the abstract ideas would obviously hold true at the absence of us or any other subject. The truth of the Law of identity, "A is A", does not depend on what A is the subject and his qualia, the material world or simply nothing. The very sense of logical laws makes it evident that subjective qualia are not their only content. This makes solipsism impossible in its extreme form subordinating the truth of the laws of logic to the existence of subject that conceives them. Here, there is an undoubted difference between object as an inner content of logic principle and subject as a specific form of qualia highlighting this content.

Logic is a formal basis for mathematics, which itself is a basis for all scientific knowledge. Thereby, qualia give us knowledge about the mathematical structure of the external material world, which itself is beyond our direct experience. The question is what can be cognized with the help of qualia besides the pure mathematical structure and relations. Is it possible to be aware of some qualities of the external world? Do some qualia, such as qualia of extension, include these qualities as their inner aspects, which could be abstracted from them? I shall discuss this in the following section. 


\subsection{The Existence and Structural Order of Qualia Can't Be Logically Deduced from Qualia Themselves and Require External Founding by Matter}

If all that we observe directly, by our senses or with a help of machinery, is a complex multitude of qualia, how is it possible to know anything about the external world?

As well as in the previous case, the answer lies in the very subjective nature of qualia. There is no relation between subjective phenomena that can be strictly deduced from them or from any other phenomenon. For example, when we see two spots, blue and cyan, neither their form nor their color likeness directly causes their proximity in the field of vision. The spatial order of objects we observe obeys to some laws of physics and geometry, however, the sensual images themselves are not ordered by any intrinsic laws. Qualia and their structures are essentially "atomic": they can assemble in different ways; there is no intrinsic necessity of their ordering in mind. In addition, there is no obvious cause for them to exist: we think about them as being possible to be and not to be. As the structure of phenomenal world can't be explained from inside, we need something external to arrange it with all its forms, aspects, and properties. If we call this external mediator a physical cause or matter, the two attributes of matter we should postulate:

1. Material events form an inherently inseparable multitude: all their real and potential relations are founded by themselves and can't differ within the given material world.

2. The relations between material events are the basis for relations of qualia mediating the specific arrangement of qualia in mind.

This automatically means that the structure of qualia and their properties is equal to the structure of material causes: each relation that can't be founded within the phenomenal world should have the equivalent intrinsic relation in matter. The opposite is also true, since each material structure capable to found qualia of specific kind must cause them, and the absence of qualia is a particular manifestation of psychophysical law. The only alternative is that some relations are accidental; however, this is unlikely in the case of the common laws and regularities. It is still a question whether matter itself is caused by some "higher" entity, or its existence is only an accident, or its nonexistence is metaphysically impossible.

It should be emphasized that the qualitative nature of matter is beyond our experience, as there is nothing in it explaining the psychophysical connection and the nature of interaction between material things. An illustration of the latter is that we can't vividly imagine how space and time interact to form the united space-time continuum. Therefore, what we call time and space is only our subjective mode to perceive the different aspects of physical reality; Immanuel Kant, who distinguished phenomena and noumena, firstly expressed this idea. The spatially extended particles and physical fields are often considered the substance of matter, but in fact, the spatial images only reflect some structural order of physical events, which is more easily observed for the relatively simple and universal extended phenomena than for the complex sensory experiences. The material causes seem to be organized as some kind of extended continuum, however, neither its intrinsic qualitative nature nor the mode of existence - as substance or some properties or relations between the material entities - is known to us. "The inverse square law is an account of gravity, but it does not show why bodies have to have gravitational attraction [10]".

All that we know about matter is its structure formed by more simple structures with specific order of members. Each of them is somehow represented in our mind, e.g. water as a transparent liquid medium and water as a complex system of small moving particles connected by molecular forces. The physical properties of liquid are reduced to the properties of its molecular structure, but it is impossible to directly reduce the sensible image of liquid water to the images of small particles. Only the abstract structure of mathematical relations can be strictly deduced from the other relations. As Searle states, consciousness is causally, but not ontologically reducible to neurobiological processes, as it has a "firstperson ontology [11]". At the same time, not qualia themselves are causally interconnected but material causes represented by them, as well as qualia to their material causes.

Hence, there is no sense to reduce qualia to any of the brain processes in space and time replacing the true material causes by their phenomenal representations. All speculations about qualitative semblance of qualia and their physical causes are groundless. To cause a quale of blue, matter shouldn't be blue itself, but it should have some essential and intrinsic relation to blue. However, this relation can't be something like the colors similarity, such as that for blue and cyan. Possibly, the inner nature of matter and psychophysical law can be conceived somehow with the help of qualia, likewise the objective logical principles. However, this kind of experience is completely different from all that contemporary science can give to us. The most it can do is to find out a tight association between the consciousness and some specific process in brain linking the appearance of qualia with the peculiar organization of physical events.

\subsection{There Is an Intrinsic Accordance Between the Qualitative and Structural Aspects of Qualia and Matter}

As we have seen, if we properly understand what we call matter, it is impossible to mix up qualia and their material causes. There is no need to prove that qualia themselves are not physical processes in brain, because we defined "physical" as the base for necessary relationship between qualia, which is obviously external to any subjective qualities. This does not mean that qualia are some kind of spiritual objects: matter causes them and seems to be their substantial basis. However, it tells us nothing about the functions of qualia and their causal efficacy. How do they influence the brain processes and behavior? Aren't they epiphenomenal properties of matter that simply accompany some physical processes in brain?

A few words should be said about the idea of causal 
connection per se. If we assert that one thing or property causes the other that supervenes on it, what does it mean? For the mind-body supervenience it has been stated: "Whenever something has a mental property, $\mathrm{M}$, at $t$, it does so in virtue of the fact that it has, at $t$, a physical base property, $\mathrm{P}$, where $\mathrm{P}$ necessitates $M$ [12]". $M$ is impossible without $P$ and changes in $\mathrm{M}$ necessarily accompany changes in $\mathrm{P}$. So how can we define one of them as the "cause" or "effect" in any sense but the order of its cognition by us? Qualia and their structure are not founded by themselves, so we presume something beyond our mind that can do it, but it tells us nothing about the possibility of matter to exist without qualia and vice versa. The metaphysical "preference" of matter is that material cause is a basis for the relations between qualia; however, it does not make qualia insignificant for causal connection.

Hence, there is no reason to consider qualia and matter separately when we think about their causal efficacy: neither qualia occur without specific material processes in brain, nor these processes seem to exist without subjective phenomena. Is it possible to construct an exact functional copy of brain without qualia? Maybe yes, maybe no, in any case, it would not be the human brain. The question is whether qualia have any particular cognitive function except allowing the human brain to exist. There may be no answer at all, but if we try to find it, the only way is to connect the phenomenal qualities with their specific structure, which might functionally organize the mental processes in brain.

On the whole, there is a deep interrelation between qualitative and structural aspects of things. The extensive line segment can exist only as an infinite multitude with continuum of parts: the quality of extension realizes a specific type of mathematical order. The similar is true for material events, which seem to be inseparably interconnected. On the contrary, qualia do not form an inseparable unity: they are somewhat like Leibniz's monads, quasi-independent in their being from each other but harmonically organized as a complicated multifold system. Each quale is an element of the entire qualitative unity, hierarchically joining senses, perceptions, and abstract ideas. As it has been mentioned above, this mode of ordering is tightly connected with the fundamental properties of qualia - phenomenology and intentionality.

The very idea that subjective phenomena innerly form the complex multidimensional qualitative space founded by similarity and dissimilarity relations is not original. According to Kristjan Loorits, qualia are "something compositional with internal structures that fully determine their qualitative nature [13]". A single quale, e.g. the red color, is not essentially atomic but includes multiple aspects; each of them remains unconscious until we begin to perceive them like something specific and separate. The quale is the meaning of some inner state in the focus of subjects' attention. There is nothing in qualia that can't be expressed in the terms of structure. The author concludes that qualia can be identified with certain neural activity patterns in brain. Again, we see an attempt to substitute structure in place of quality, though both of them are in fact specific and inseparable aspects of mental phenomena.

Thus, it is a great error to consider qualitative aspect insignificant, only occasionally associated with this or that type of structural organization. This may be the main reason for mistakes about qualia. If we think consciousness to be simply a computational process in brain, as functionalists do, we should remember Searle: a computation is defined syntactically, but logical syntax in not sufficient for semantic content [14]. If we argue like Dennett that all qualitative semantic can be expressed in the pure languages of mental dispositions, will it abnegate qualia? Surely, it will not, as subjective qualities are that makes such structure of dispositions to exist. The qualitative essence itself can't be expressed by any words; however, it founds the whole system of relations in subjective world. We can't change any quale without influencing its relation to the others, but this only affirms the basic principle: a certain set of qualities $-a$ certain structural order.

This fact does not contradict to my previous statement that the order of qualia is founded by matter. This has been supposed a priori for relations that can't be founded within subjective world, but not for inner relations, such as intentionality, which is inseparable from subjective world in any of its manifestations. Physical causes only forward the subjective intentionality in one or the other way; they are neither equal to it nor can functionally substitute it in thinking process. The last I shall discuss in the following section.

\subsection{The Chief Cognitive Function of Qualia Is to Abstract the Relations and Realize the General Properties}

When we speak about psychophysical connection, there is no need to consider it unidirectional. Not only matter realizes qualia, but the inner structure of qualia also can be somehow reflected by the structure of material events. We do not observe such kind of interaction in simple physical systems, in which the principle of causal closure is strictly fulfilled, but as well, we do not observe qualia there. Hence, there is no reason to defend this principle at all costs for the psychophysical systems. Qualia, not matter, is what we directly experience, thus, it's contradictory to deny the existence of qualia or their possible efficiency simply to please some materialistic conceptions. Moreover, as the nature of material space and time is unknown to us, there is no sense to assert that physical matter is intrinsically "extended" and thereby it can't interact with the "conscious" mind.

The mode of psychophysical interaction is different from any relations we observe in phenomenal world - it can't be imagined, but this doesn't mean that it can't exist. It shouldn't be considered the "vital" force, because all the vital biological processes in brain are reduced to physical interactions, whereas phenomenal qualia can't be reduced to them. The association of qualia with brain, a complex biological system, is rather explained by the evolution process that makes brain possible to produce qualia giving it some functional advantages [15]. 
Thus, the possible functional role of qualia in mental processes doesn't contradict to any laws of nature. Probably, someday we shall succeed in demonstrating how the "natural" physical processes in brain change in connection with qualia. However, this would not explain why subjective phenomena at all are needed for thinking. What in qualia makes them possible facilitators or accelerators of mental activity?

Imagine the idea of an abstract law, e.g. " $\mathrm{A}$ is $\mathrm{A}$ " or " $\mathrm{A}$ is not B". This idea is somehow represented in the form of brain activity: specific pattern of neurons firing, the order of impulses in neural tracts, etc. The structure of these processes should involve some kind of circular reference representing the meaning of equality of a thing to itself. We conceive this law as absolutely clear and strict without any exceptions. However, what could be the material substrate for such a common relation? If A and B are undoubtedly different, how can they be represented by the brain functional units? There are no exact borders between the neuronal networks or neurons, which are the complicated atomic structures: all the boundaries of material objects can be drawn only approximately. There may be some obscure physical properties, which indubitably draw these boundaries, but at present time we know nothing about them, and if we knew them, I state, they would likely be linked to qualia.

All the objects, such as table and floor and even table and human brain, are strictly separated in our mind, not in reality, though their material structures are quite different. Table can't think and analyze, brain can do this due to the specific order of its elements. The activity of neurons makes it possible to define the main characteristics of the sensory signals and to make conclusions about their properties. The brain activity is somewhat like the potting of balls in billiards: image or thought is "caught" by some neural processes and "kept" by them. The function of brain is to direct the stream of thoughts sorting them according to their form. However, this sorting only outlines the contours of things, ideas, and notions: they are never pure in their neurophysiological form. This is the main problem of artificial intelligence: machine can analyze, it can abstract the general principles and laws, but it never understands the exact meaning of them.

Is not such a blind mind clever enough to grasp all the information about things and their properties? Indeed, it can successfully solve some practical and theoretical problems, but it must be feeble, if understanding the necessity of the common principles is required. Something particular happens in our mind, when we think A is A. Plato's concept of ideal forms appears to be true in a certain way, when we speak about consciousness: we don't possess the ideas estranged from any raw senses and perceptions, but the ideas in mind form a quasi-separated notional layer representing general properties and relations between the observed objects. We cognize common through concrete and the abstract relations through the concrete things. This is a specific type of intentionality, peculiar to qualia: they highlight the meanings, properties, and general laws, which are only outlined by the physical processes in brain. Every quality realizes a specific type of relations, and some subjective qualities realize the abstract properties being the aspects of sensual perceptions.

Let us return to our example with "left and right". These characteristics are relative in the material world, but they are definite in the world of qualia. Galileo's principle of relativity doesn't hold in the subjective world: qualia "absolutize" the relative properties. In addition, contrary to the physical world, we see a certain discontinuity in the world of qualia: the borders of objects, the different sensory modalities, the difference between abstract ideas and concrete things, etc. Hence, phenomenal structure is not a complete copy of the material one: there are no physical analogues for the objects' forms and boundaries, as well as for the abstract ideas. The psychophysical interaction is a kind of retranslation, where the continuous or quasi-continuous physical processes are unambiguously transformed to the pattern of discrete mental forms and ideas. All the "gaps" between sensory modalities, senses and abstract thoughts, etc., seem to be predefined somehow by the brain processes organization [15]. However, a unique feature of qualia structure is that its different aspects and properties are qualia themselves; this type of order is impossible for matter where the real things ontologically differ from the general properties and found them. Intentionality of qualia simply reflects their specific mode of existence - we can call it a circular foundation: here, an abstract idea may become the medium by which we perceive a concrete thing, and vice versa.

This all makes qualia the potent intermediates in the cognition and decision-making activity. We don't know exactly how qualia can influence the brain processes: their specific order in its turn may be reflected by the structure of neural processes or additionally stabilize a given structure compared to others. The special properties of qualia distinctness or irrevocability, qualitative diversity, and connection to memory - may help the brain to elaborate the optimal behavioral strategy [16]. Qualitative consciousness is tightly connected to process of attention that seems to play a role of selector, choosing one or another behavioral program to perform [17]. Self-awareness and consciousness are tightly connected to frontal lobes [18], as well as to some other cortex and subcortex structures. Here, the main task is to find the physical or physiological process in brain that is minimally sufficient to generate qualia [19].

One of the most promising theories for consciousness is so-called integrated information theory (IIT) [15]. According to IIT, consciousness is a "fundamental property possessed by physical systems, which have specific causal properties". Namely, it should be a definite, integrated, structured system, which elements can be causally composed in various combinations, forming interdependent subsystems with a developed feedback connections. A cause-effect structure maximally irreducible to the others is a "concept", and a structure composed of concepts is a "conceptual structures", which is considered equal to quale. According to the authors, this structure "completely specifies both the quantity and the quality of experience". The undoubted strengths of IIT are that it: 1. deduces its postulates from the structural properties of qualia; 2 . introduces a specific parameter for system that 
must have qualia - the local maximum of integrated information; 3. agrees with experimental data; 4. predicts what system should be conscious and what should not be, e.g. why qualia are associated with the cerebral cortex but not with the cerebellum or personal computers, or virtual consciousness simulation, or America as a community of Americans. Information integration in brain considers the main function of qualia. To my mind, the only omission of IIT is not to state the inner relation between qualitative and structural aspects in general - that's exactly what I want to show in this article.

If we replaced the physical elements of the brain with some others or simply modelled the brain processes using known physical equations, without any qualia, what would change in a subject's behavior? Would some mental tasks become more difficult for subject in the absence of qualia? Yes, I state, though it does not mean that it would be impossible for him to "blindly" solve these tasks. Probably, his brain should be more complex to provide the functions of speech, abstract thinking, and even perception: in all these processes qualia seem to be deeply involved. Undoubtedly, he would completely lose the ability to assign emotional values to events and his own deeds. Some formal algorithms of evaluation could effectively substitute them, but this would have nothing common with the true emotions except some functional similarity. Our zombie would presumably fail trials on understanding and creative usage of basic laws, both abstract and moral. This is a great problem of artificial intelligence: whether the benefits of using it to solve some tasks justify the risks connected to the possible complete absence of humanity and moral sense.

\section{Conclusion}

Qualia and matter form a functional unity, participating together in the process of cognition. Being the different aspects of the cognitive process, qualia and their physical causes are tightly interrelated. We do not know whether matter is possible without qualia or not. We do not know as well which physical processes in brain are untimely related to qualia, though an undoubted progress has been made here [15]. All that we can say about the psychophysical problem it is as hard as all the problems regarding the basic natural laws and can't be finally resolved by any empirical science. Some types of qualia, i.e. sensory qualities, are too complex for the direct analysis of their structure, whereas the "extended" qualia of processes running in space and time are much simpler to abstract the basic physical principles, which define their structure. Raw senses and "extended" qualia are the two sides of the same coin, and the substance of the coin - the inner nature of matter - is unknown.

To speak about the possible function of qualia, we should consider their qualitative peculiarity that ontologically founds their specific structure and, in turn, may organize the brain processes. The chief function of qualia seems to be the formation of the hierarchical system of relations where common and abstract are specifically outlined, represented as the "floors" above the "ground" of the pure senses. This can be fruitfully used by analytical processes in brain which otherwise would seek the common rules and laws "by touch". To rephrase the famous expression: brain without qualia is blind and qualia without brain are dead. Biological brain is a kind of qualitative computer, not simply guessing the laws of the world, but doing it in compliance with the very sense of them.

This is, I believe, the way to solve the "hard problem" of consciousness. There is no way to realize or understand anything but realize or embody it as specific phenomenon: qualia make it possible to conceive the abstract laws and principles in pure form. Not only they give the form to these principles but also make them evident placing them under the "light of reason". This is not simply a metaphor, as it expresses the intimate relation between the main attributes of qualia - intentionality and self-evidence.

Does anything but qualia possesses the same structure, something that could substitute them in brain? This is the general question about quality-to-structure relation: is there a one-to-one correspondence between them or the same structures can be realized by different qualities, or some types of structure could not exist basically? Some philosophers believe that a specific structure of material process is sufficient to generate qualia [15], hence we do not need to include them separately. Those who think the structure of consciousness completely substitute qualia shouldn't stop here, as exactly the same can be said about the physical world. To develop Dennett's idea: not only all humans are zombies without any qualia, but the whole world is nothing more than some kind of mathematical relations without any material substance. It's tempting to deduce all things with their structure and qualities from the pure laws of logic and math. However, the absolute evidence of qualia existing as specific phenomena is in contradiction to this theory.

Anyway, we are still far from making artificial intelligence that would be capable of understanding the very essence of the abstract tasks. Though machine may become even more analytically powerful than human brain, its mode of analysis remains a complicated trial and error method, and its logic the logic of the blind watchmaker. The root of the problem is ontological: I need qualia or something like them to become a sighted person.

\section{Acknowledgements}

I would like to thank Vsevolod Konnov for fruitful and comprehensive discussions of the problem of qualia.

\section{References}

[1] D. J. Chalmers. Facing up to the Problem of Consciousness, in Journal of Consciousness Studies, Volume 2, Issue 3, 1995, pp. 200-219.

[2] J. R. Searle. Intrinsic Intentionality, in Behavioral and Brain Sciences, Volume 3, 1980, pp. 450-456. 
[3] M. M. Stanciu. The Explanatory Gap: 30 Years after Procedia, in Social and Behavioral Sciences, Volume 127, 2014, pp. 292-296.

[4] D. Dennett. From Bacteria to Bach and Back: The Evolution of Minds. W. W. Norton \& Company, Inc., 2017.

[5] F. Jackson. (1982) Epiphenomenal Qualia, in Philosophical Quarterly, Volume 32, 1982, pp. 127-136.

[6] P. Churchland. Eliminative Materialism and the Propositional Attitudes, in the Journal of Philosophy, Volume 78, Issue 2, 1981, pp. 67-90.

[7] D. C. Dennett. Instead of Qualia, in Brainchildren. Essays on Designing Minds, Cambridge, MA: MIT Press, 1998.

[8] D. C. Dennett. The Unimagined Preposterousness of Zombies, in Brainchildren. Essays on Designing Minds, Cambridge, MA: MIT Press, 1998.

[9] G. Graham, T. Horgan, J. Tienson. Phenomenology, Intentionality, and the Unity of Mind, in the Oxford Handbook of Philosophy of Mind, Oxford NY: Oxford University Press, 2009.

[10] J. Searle. The Rediscovery of the Mind, in a Bradford Book, First MIT Press P., 1994.

[11] J. R. Searle. Why I am not a Property Dualist, in Journal of Consciousness Studies, Volume 9, Issue 12, 2002, pp. 57-64.
[12] J. Kim. Mental Causation, in the Oxford Handbook of Philosophy of Mind, Oxford NY: Oxford University Press, 2009.

[13] K. Loorits. Structural Qualia: a Solution to the Hard Problem of Consciousness, in Frontiers in Psychology, Volume 5, 2014, doi: 10.3389/fpsyg.2014.00237.

[14] J. R. Searle. (1993) the Problem of Consciousness, in Social Research, Volume 60, Number 1, 1993, pp. 3-16.

[15] G. Tononi, C. Koch. Consciousness: Here, There and Everywhere? In Philosophical Transactions of the Society B Biological Sciences, Volume 370(1668), 2015, doi: 10.1098/rstb.2014.0167.

[16] V. S. Ramachandran, W. Hirstein. Three Laws of Qualia, in Journal of Consciousness Studies, Volume 4, Issue 5-6, 1997, pp. 429-458.

[17] J. J. Prinz. A Neurofunctional Theory of Consciousness, in Philosophy and Neuroscience. Brook A \& Akins K (Eds.). Cambridge University Press, 2003.

[18] R. Carter. Mapping the Mind. University of California Press, Rev Upd edition, 2010.

[19] D. J. Chalmers. What is a Neural Correlate of Consciousness? In Neural Correlates of Consciousness: Empirical and Conceptual Issues, MIT Press, 2000. 